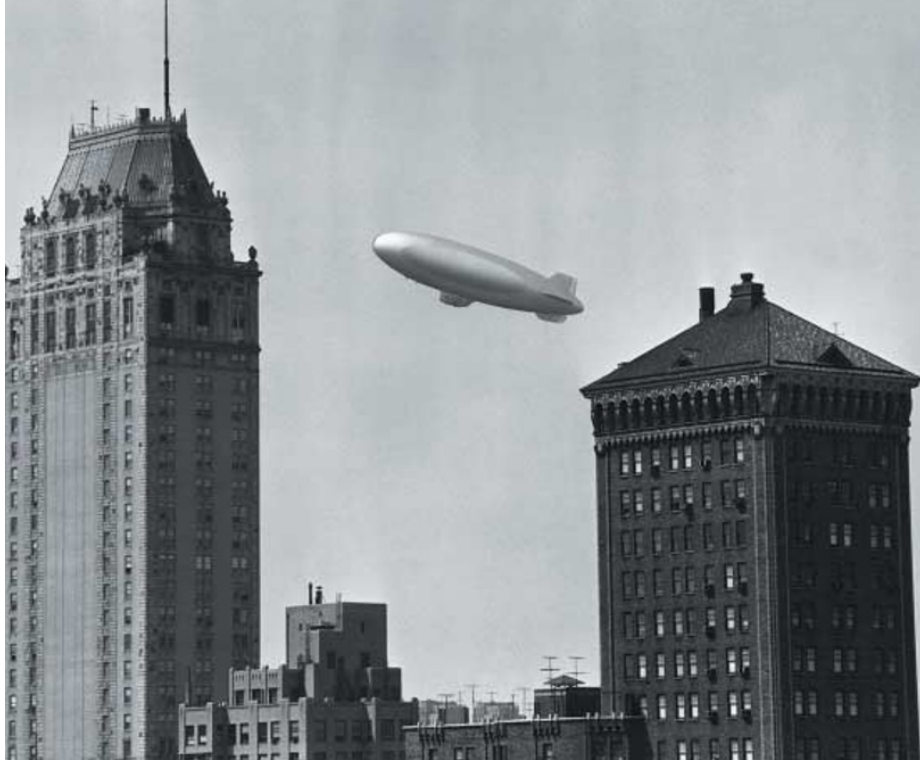

B-CELL DEVELOPMENT

\section{Plasma-cell differentiation requires Blimp1}

Although the transcriptional repressor B-lymphocyte-induced maturation protein 1 (Blimp1) has been previously shown to induce B-cell differentiation into immunoglobulinproducing plasma cells, until now, it has not been identified as non-redundant and essential for this process.

Mice lacking the gene encoding Blimp1, Prdm1, die in utero, so to study the dependence of B-cell differentiation and function on Blimp1, Shapiro-Shelef et al. generated mice in which $\operatorname{Prdm} 1$ was specifically eliminated in mature B cells, $\operatorname{Prdm} 1^{\text {flox/flox }} \mathrm{CD} 19^{\text {cre/+ }}$ mice. B-cell development was normal in these animals but serum immunoglobulin was markedly reduced, even in unimmunized mice.

After immunization with either a thymus-independent (TI) or thymusdependent (TD) antigen, the immunoglobulin response generated in $\operatorname{Prdm} 1^{\text {flox/flox }} \mathrm{CD} 19^{\text {cre/+ }}$ mice was substantially diminished when compared with control animals, as was immunoglobulin secretion after re-challenge with TD antigen. This decrease in immunoglobulin production correlated with a marked reduction in the number of antigen-specific immunoglobulin-secreting cells and consistent with this, few plasma cells or preplasma memory B cells could be detected in $\mathrm{Prdm} 1^{\text {flox/flox }} \mathrm{CD} 19^{\text {cre/t }}$ mice after either primary or secondary exposure to TD antigen. So, Blimp1 is required for the development of both plasma cells and pre-plasma memory B cells during both a primary and memory response.

To investigate the mechanisms by which Blimp1 regulates immunoglobulin secretion and plasma-cell differentiation, Shapiro-Shelef et al. analysed Blimp1-deficient B cells that were exposed to lipopolysaccharide (LPS) ex vivo. In the absence of Blimp1, switching from the membrane-associated form of the immunoglobulin heavy chain $(\mu \mathrm{M})$ to the secreted form $(\mu \mathrm{S})$ was markedly impaired, as was the induction of $\mu \mathrm{S}$ messenger RNA. In addition, the transcription factor X-box binding protein 1 (XBP1) was not induced by LPS stimulation of $\mathrm{Prdm}^{-1-} \mathrm{B}$ cells, identifying it as a downstream target of Blimp1. However, retrovirus-mediated overexpression of XBP1 by Blimp1deficient $B$ cells was insufficient to rescue plasma-cell development, indicating that Blimp 1 must target many factors to induce plasma-cell differentiation.

These studies using a conditional gene-deletion strategy define Blimp1 as essential for the differentiation of plasma cells and immunoglobulin secretion, and indicate that this transcription factor also has a key role in pre-plasma memory B-cell development.

Karen Honey

(2) References and links ORIGINAL RESEARCH PAPER Shapiro-Shelef, M. et al. Blimp-1 is required for the formation of immunoglobulin secreting plasma cells and preplasma memory cells. Immunity 19, 607-620 (2003)

WEB SITE

Kathryn Calame's lab: http://cpmcnet.columbia edu/dept/gsas/biochem/faculty/calame.html

\section{IN BRIEF}

\author{
CYTOKINES
}

IL-23 produced by CNS-resident cells controls T-cell encephalitogenicity during the effector phase of experimental autoimmune encephalomyelitis.

Becher, B., Durell, B. G. \& Noelle, R. J. J. Clin. Invest. 112, 1186-1191 (2003)

Interleukin-23 (IL-23) consists of a unique p19 subunit and the p40 subunit of IL-12, and is crucial for the development of experimental autoimmune encephalomyelitis in mice. Becher et al. use p40-deficient mice to generate irradiation bone-marrow chimaeras and show that in the absence of $\mathrm{p} 40$ expression by irradiation-resistant cells, previously activated antigen-reactive cells infiltrate the central nervous system (CNS), but do not induce encephalitogenicity. In addition, disease induced by immunization is markedly diminished when $\mathrm{p} 40$ is absent from irradiation-resistant cells and this is associated with a bias towards the production of Thelper 2-type cytokines by infiltrating cells. The authors, therefore, suggest that $\mathrm{p} 40$ expression by CNSresident cells contributes to T-cell polarization and thereby modulates disease pathogenesis.

\section{TRANSPLANTATION}

\section{Prevention of organ allograft rejection by a specific} Janus kinase 3 inhibitor.

Changelian, P. S. et al. Science 302, 875-878 (2003)

The development of effective immunosuppressive agents that are targeted specifically to immune cells and do not produce serious side effects remains a pressing need for the prevention and treatment of transplant rejection. This study reports pre-clinical trials of a new immunosuppressive agent (CP-690,550) that prolonged survival in both a mouse model of heart transplantation and a cynomolgus monkey model of kidney transplantation, but that did not result in the dose-limiting side effects that are associated with present therapies. CP-690,550 inhibits Janus kinase 3 (JAK3), which is crucial for signalling through cytokine receptors that use the common $\gamma$-chain and, therefore, for development and homeostasis of immune cells. It is the first JAK3 inhibitor to have shown efficacy in non-human primates.

\section{IMMUNOTHERAPY}

\section{A critical role for OX40 in T cell-mediated immunopathology during lung viral infection.}

Humphreys, I. R. et al. J. Exp. Med. 198, 1237-1242 (2003)

Respiratory infections are an important example of how an overactive T-cell response can be more of a hindrance than a help, with T-cell responses resulting in airway occlusion and pathology. Previous T-cell-targeted therapies have affected both bystander and antigen-specific T cells, which might result in dangerous immunosuppression. These authors used an OX40-immunoglobulin fusion protein to target recently activated $\mathrm{T}$ cells specifically. OX40 signalling prevents the death of activated $\mathrm{T}$ cells, but it is not expressed by naive $\mathrm{T}$ cells. Interference with this T-cell survival signal in a mouse influenza model reduced immunopathology without preventing virus clearance. 